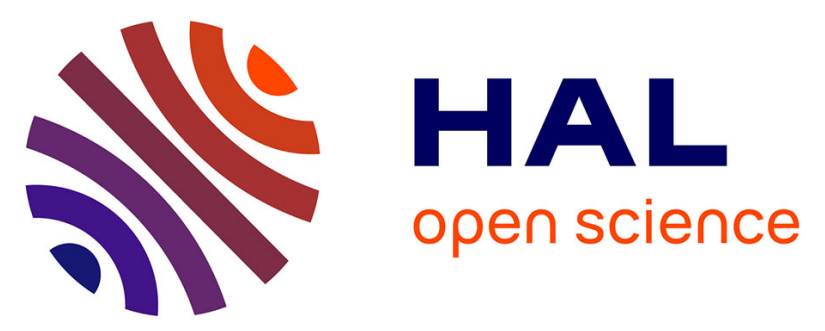

\title{
Energy scaling of a nonlinear compression setup using passive coherent combining
}

Florent Guichard, Yoann Zaouter, Marc Hanna, Franck Morin, Clemens

Hönninger, Eric Mottay, Frédéric Druon, Patrick Georges

\section{To cite this version:}

Florent Guichard, Yoann Zaouter, Marc Hanna, Franck Morin, Clemens Hönninger, et al.. Energy scaling of a nonlinear compression setup using passive coherent combining. Optics Letters, 2013, 38 (21), pp.4437. 10.1364/OL.38.004437 . hal-00925384

\section{HAL Id: hal-00925384 \\ https://hal-iogs.archives-ouvertes.fr/hal-00925384}

Submitted on 21 Apr 2016

HAL is a multi-disciplinary open access archive for the deposit and dissemination of scientific research documents, whether they are published or not. The documents may come from teaching and research institutions in France or abroad, or from public or private research centers.
L'archive ouverte pluridisciplinaire HAL, est destinée au dépôt et à la diffusion de documents scientifiques de niveau recherche, publiés ou non, émanant des établissements d'enseignement et de recherche français ou étrangers, des laboratoires publics ou privés. 


\title{
Energy scaling of a nonlinear compression setup using passive coherent combining
}

\author{
Florent Guichard, ${ }^{1,2, *}$ Yoann Zaouter, ${ }^{2}$ Marc Hanna, ${ }^{1}$ Franck Morin, ${ }^{2}$ Clemens Hönninger, ${ }^{2}$ \\ Eric Mottay, ${ }^{2}$ Frédéric Druon, ${ }^{1}$ and Patrick Georges ${ }^{1}$ \\ ${ }^{1}$ Laboratoire Charles Fabry, Institut d'Optique, CNRS, Université Paris-Sud, 2 Ave. Augustin Fresnel, 91127 Palaiseau Cedex, France \\ ${ }^{2}$ Amplitude Systèmes, 11 avenue de Canteranne, Cité de la Photonique, 33600 Pessac, France \\ *Corresponding author: florent.guichard@institutoptique.fr
}

\begin{abstract}
Passive spatial and temporal coherent combining schemes are implemented to scale the output energy of a nonlinear temporal compression setup. By generating 32 replicas of the incident femtosecond pulses, the output of a highenergy fiber chirped-pulse amplifier can be compressed using self-phase modulation in a large-mode-area rod-type fiber at peak-power levels well beyond the self-focusing power. We demonstrate the generation of $71 \mathrm{fs} 7.5 \mu \mathrm{J}$ pulses at $100 \mathrm{kHz}$ repetition rate, corresponding to a peak power of $86 \mathrm{MW}$.
\end{abstract}

Nonlinear postcompression is a well-established technique to reduce the pulse duration of high-power, highenergy femtosecond laser systems. In such systems, the incident laser field is strongly confined in a solid-core fiber, a Kagome-type hollow-core fiber, or a gas-filled capillary that preserves a high intensity over an extended distance, and allows large spectral broadening via selfphase modulation (SPM). Suppression of the induced chirp produced by the nonlinear interaction in the medium with appropriate dispersive elements then leads to pulse shortening. However, these systems are limited in output energy by various effects such as self-focusing, optical damage, or gas ionization. These phenomena limit the scalability of the above-mentioned approaches to the microjoule level when using fused-silica fibers [1] and to the few-millijoule level [2] for capillary setups. The scalability and robustness of setups based on Kagome-type fibers are currently subject to promising research, and postcompression of a $\sim 0.5 \mathrm{~mJ}$ pulse to $50 \mathrm{fs}$ was recently demonstrated [3]. Nevertheless, despite the strong potential of such Kagome hollow-core fibers to support the propagation of even higher-energy pulses, previously mentioned limitations, in particular ionization, are still expected.

Coherent combining concepts have recently been used to scale the energy of ultrafast systems in the framework of optical amplifiers. In particular, passive implementations have been used, consisting in spatially $[4,5]$ and/ or temporally [6] redistributing the pulse energy into several independent replicas. This decreases the peak power inside the amplifier, thereby allowing energy scaling, before passive recombination to reveal the total output pulse energy. Following this approach, we recently reported a passive coherent beam combining chirped-pulse amplifier that uses a Sagnac interferometer (SI), allowing the generation of $300 \mathrm{fs} 650 \mu \mathrm{J}$ pulses at $92 \mathrm{kHz}$ from two rod-type fibers [7]. We also demonstrated a stretcher-free spatio-temporal divided-pulse amplifier experiment generating $3.1 \mu \mathrm{J} 50 \mathrm{fs}$ pulses [8], highlighting the potential of coherent combining in the nonlinear regime with the B-integral largely exceeding 20 rad and its compatibility for the generation and preservation of broadband spectra.

In this Letter, we propose to use, in a proof-of-principle experiment, a passive spatial and temporal divided-pulse coherent combining scheme to scale the energy of the nonlinear pulse-shortening setup. Up to 32 replicas are generated from a high-energy fiber chirped-pulse amplifier (FCPA). These replicas are propagated into a piece of undoped rod-type fiber that introduces SPM and dispersion to obtain sub-100-fs pulses after compression. This allows us to go well beyond the $4 \mathrm{MW}$ peak power limit associated with self-focusing in fused silica, and generate $71 \mathrm{fs}$ and $7.5 \mu \mathrm{J}$ pulses, corresponding to a measured peak power of $86 \mathrm{MW}$. The system efficiency is studied as a function of increasing $2^{N}$ number of replicas, revealing a weak dependence on the number of temporal division stages $N$. These results represent, to our knowledge, the first use of coherent combining outside the context of optical amplifiers, and show that it can be used in any energy-limited optical setup, including systems that manipulate sub-100-fs pulses.

The experimental setup is depicted in Fig. 1 and starts with a transform-limited $<400$ fs high-energy FCPA. This system can deliver up to $300 \mu \mathrm{J}$ pulse energies in a diffraction-limited output beam at a repetition rate of $400 \mathrm{kHz}$. It is followed by the compact $(30 \mathrm{~cm} \times 60 \mathrm{~cm})$ postcompression setup. Time divisions are implemented by means of four highly birefringent a-cut yttrium vanadate $\left(\mathrm{YVO}_{4}\right)$ crystals alternatively aligned at $45^{\circ}$ and $0^{\circ}$ from the incident linearly polarized beam. Each crystal has a length doubled from the previous one in order to generate equally separated pulses. This allows the generation of 16 orthogonally polarized time-delayed replicas of the incident pulse with a minimum delay of $1.95 \mathrm{ps}$, given by the smallest crystal length of $2.5 \mathrm{~mm}$. In this configuration, the delay between two adjacent replicas is sufficient to avoid significant temporal overlap that could lead to nonlinear coupling between successive replicas and imperfect recombination. The replica train passes through a Faraday rotator and a quarter waveplate oriented at $45^{\circ}$ and enters the SI, which generates two counterpropagating 


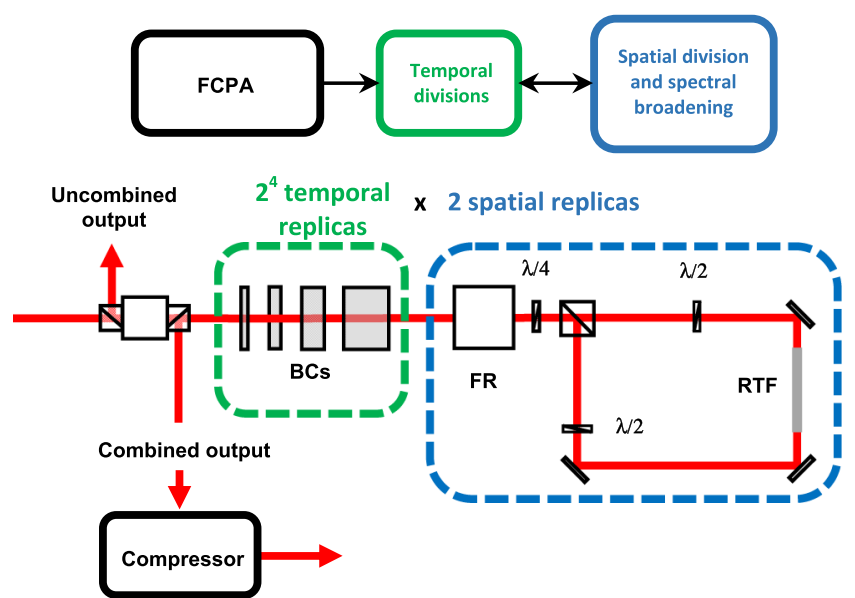

Fig. 1. Experimental spatio-temporal $30 \mathrm{~cm} \times 60 \mathrm{~cm}$ postcompression setup. BCs, birefringent crystals; FR, Faraday rotator; RTF, rod-type fiber.

beams, each containing 16 temporal replicas, totaling 16 (temporal) $\times 2$ (spatial) $=32$ replicas inside the rodtype fiber. The quarter waveplate together with the SI act as a neutral element for the replicas polarization states since the SI behaves as a half-waveplate oriented at $45^{\circ}[8]$. Large spectral broadening occurs in an 8-cm-long undoped rod-type fiber with a mode field diameter of $45 \mu \mathrm{m}$. The experimental coupling efficiency to this fiber, measured using a pinhole at the output, is typically $~ 85 \%$. A set of half-waveplates on each side of the Sagnac loop, together with the Faraday rotator and the quarter waveplate, ensures reciprocal propagation backward in the vanadate crystals to perform coherent combination. Indeed, as the polarization state is unchanged after the SI and the quarter waveplate, the round trip in the Faraday rotator allows a $90^{\circ}$ rotation of the replica's polarization state, which finally leads to compensating the incident optical delay introduced by the vanadate crystals during the forward propagation. Finally, the output recombined pulse is extracted through the first polarizer of the optical isolator. The overall transmission of the setup, including Fresnel reflections on the uncoated rod-type facets as well as the 16 crystal interfaces and the rotator losses, was experimentally measured to be $45 \%$.

After extraction, the combined beam is sent in an $89 \%$ efficiency single-pass $1250 \mathrm{l} / \mathrm{mm}$ transmission gratingbased compressor unit. This high efficiency is achieved by the single-pass geometry, at the expense of a slight spatial chirp of $20 \mu \mathrm{m}$, which can be neglected in view of the total beam size of $2 \mathrm{~mm}$. The output energy after compression, including temporal and spatial combining contributions, is shown in Fig. $\underline{2(a)}$ as a function of energy per replica.

The system is first operated without any temporal division, corresponding to the generation of only two spatially separated replicas, in order to determine the combining efficiency of the SI setup alone. For increasing energies per replica, the spatial combining efficiency, defined as the ratio between the combined beam average power and the total output power, remains almost constant above $92 \%$. This good efficiency indicates that the system can be kept symmetrical enough with two replicas, and that the beam mismatches are low.
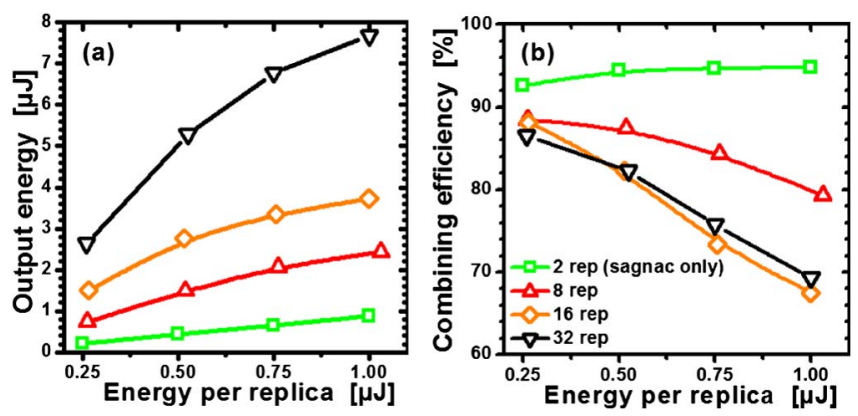

Fig. 2. (a) Output energy and (b) combining efficiency after compression deduced from spatial and temporal combining efficiencies for two spatially separated replicas (green) and after insertion of, respectively, two (red), three (orange), and four (black) vanadate crystals.

An increasing number of temporal replicas are then generated by adding crystals on the beam path before the SI. The temporal recombination steps can lead to parasitic satellite pulses in the time domain. We therefore define the temporal efficiency as the ratio of the energy contained in the main pulse divided by the total pulse energy at the output of the combining stages. The total combining efficiency, defined as the product of temporal and spatial efficiencies, is plotted in Fig. 2(b) as a function of energy per replica for an increasing number of time divisions. It decreases as the energy per replica increases, because the higher nonlinearity level makes it experimentally more difficult to equalize the phase shifts between all the replicas. These nonuniform phases turn into nonideal polarization states after the SI and finally imperfect temporal recombination and parasitic pulses, as previously observed in [8]. The total efficiency drops in the vicinity of $70 \%$ at $1 \mu \mathrm{J} /$ replica for large numbers of replicas. Output energy increases with the number of replicas to finally reach $7.5 \mu \mathrm{J}$ for 32 replicas, a record value for solid-core fiber-based postcompression setups. At this energy, the peak power inside the fiber is around 2.3 MW for each replica.

In order to study the effect of coherent combining on nonlinear pulse compression, the output pulse autocorrelation was systematically recorded for a varying amount of energy per replica and a varying number of divisions. Results are displayed in Fig. 3(a), and suggest that the pulse compression ratio is mostly independent of the number of temporal divisions performed. For an energy around $1 \mu \mathrm{J} /$ replica, the autocorrelation full width at half-maximum (FWHM) tends to a minimum owing to the usual trade-off between large spectral broadening and accumulation of uncompressed higher-order spectral phase. At this energy level, the autocorrelation FWHM is close to $90 \mathrm{fs}$ almost regardless of the number of replicas. A slight difference is observed for 32 replicas due to the difficulty in maintaining the same pulse characteristics before propagation in the nonlinear fiber. Indeed, given the small $\mathrm{YVO}_{4}$ crystal apertures, the high peak intensity before temporal divisions is sufficient to generate a nonnegligible amount of SPM and selffocusing inside the vanadate crystals, which can be only partially compensated by tuning the FCPA compressor, therefore affecting the spectral broadening and subsequent pulse compression. Nevertheless, shorter 

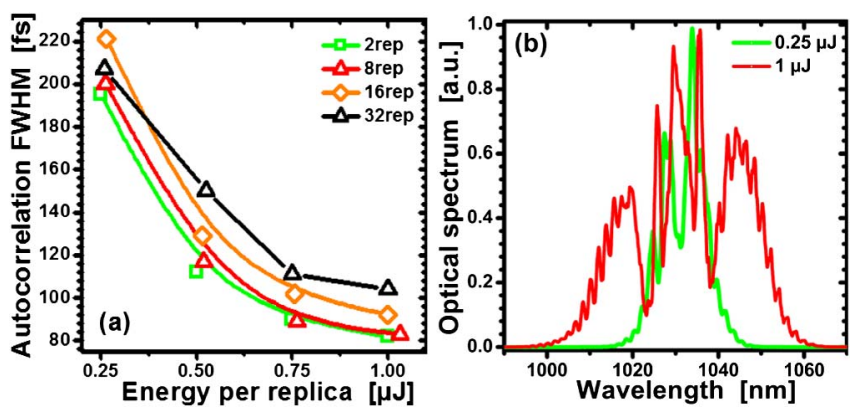

Fig. 3. (a) Autocorrelation FWHM for two spatially separated replicas (green) and after insertion of, respectively, two (red), three (orange), and four (black) vanadate crystals. (b) 32 replicas, coherently combined output spectra for $0.25 \mu \mathrm{J}$ (green) and $1 \mu \mathrm{J}$ (red) per replica.

pulsewidths could be maintained with a large count of replicas by means of larger aperture crystals and incident beam spot size. The use of free-space delay lines [9] can ultimately be required when the cross sections of the birefringent crystals become too large.

Typical SPM-induced spectra for 32 replicas at two different values of energy/replica are plotted in Fig. 3(b) to illustrate the nonlinear spectral broadening. Starting with an input FWHM of $8 \mathrm{~nm}$, it reaches $10 \mathrm{~nm}$ at $0.25 \mu \mathrm{J} /$ replica and more than $30 \mathrm{~nm}$ at $1 \mu \mathrm{J} /$ replica. The small modulations visible across the entire spectrum have a period of $\Delta \lambda=1.80 \mathrm{~nm}$, corresponding to a $1.96 \mathrm{ps}$ time delay, and can be identified as resulting from the imperfect time recombination-induced parasitic replicas.

The long-range autocorrelator is used for temporal contrast investigation. Because the delay introduced by the longest crystal is $31.2 \mathrm{ps}$, this device allows us to get a clear and complete picture of the temporal combining efficiency. We thus investigate in more detail the temporal characteristics, i.e., the temporal profiles and temporal combining efficiencies, of the output pulse at different energies. This temporal combining efficiency is dependent on the number of pulse divisions and divided-pulse energy. It is characterized by the appearance of parasitic side pulses around the main recombined pulse. For energies per replica up to $0.5 \mu \mathrm{J}$, parasitic side pulses are barely visible. As an example, the autocorrelation acquired for 32 replicas and $0.25 \mu \mathrm{J} / \mathrm{replica}$ is plotted in linear and logarithmic scale in Figs. 4(a) and 4(b). The overall energy contribution from the side pulses is less than $5 \%$, i.e., corresponding to a temporal combining efficiency around $95 \%$, and is mostly independent of the number of pulse replicas used. For higher energies, the temporal efficiency decreases to reach $80 \%$. In the highest energy case, the maximum energy fraction contained in a single parasitic pulse is $6.7 \%$. The long-range autocorrelation corresponding to a main pulse energy of $7.5 \mu \mathrm{J}$ obtained with 32 replicas is plotted in Figs. 4 (c) and 4(d) in linear and logarithmic scale.

In order to investigate the temporal profile of the pulse at the maximum energy of $7.5 \mu \mathrm{J}$ obtained with 32 replicas, single-shot second-harmonic generation frequencyresolved optical gating (SHG-FROG) measurements are carried out and compared to independent autocorrelation and spectrum measurements. The retrieved intensity profile, and the experimental and retrieved SHG-FROG
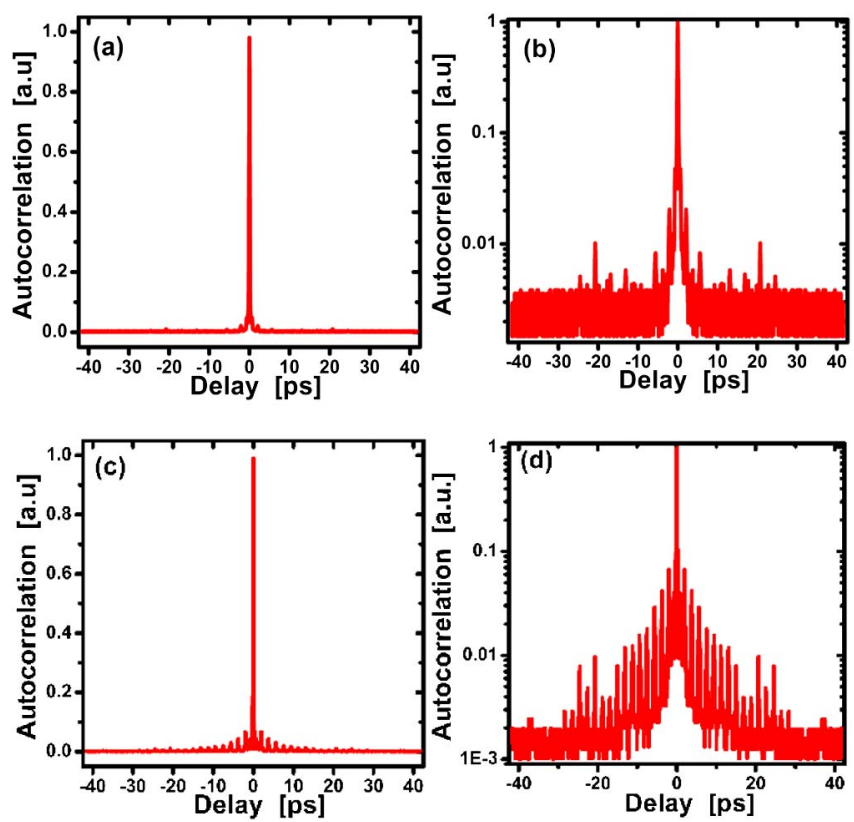

Fig. 4. (a) Long-range autocorrelation in linear scale after compression for 32 replicas at $0.25 \mu \mathrm{J}$ per replica and (b) corresponding logarithmic scale autocorrelation. (c) and (d) Linear scale logarithmic scale autocorrelation for 32 replicas and $7.5 \mu \mathrm{J}$ after compression.

trace, are shown in Figs. 5(a) and 5(b), respectively. The retrieved intensity profile exhibits a pulse duration of $71 \mathrm{fs}$ with a SHG-FROG error of $38 \cdot 10^{-4}$ on a $512 \times$ 512 grid. The retrieved spectrum FWHM is $36.2 \mathrm{~nm}$, in good agreement with the independently measured spectrum, leading to a time-bandwidth product of 0.73 . The temporal profile is characteristic of residual cubic spectral phase introduced by the compressor that could be reduced with another appropriate compression setup, such as adequate chirped mirrors. The Fouriertransform-limited pulse duration is $60 \mathrm{fs}$, indicating a satisfactory compression quality even though further improvement is clearly possible. Still, taking the FROGmeasured intensity profile into account, the measured peak power is as high as $86 \mathrm{MW}$, a record value for postcompression stages based on solid-core silica fibers, and demonstrates the advantage of the described coherent combining technique.

Further improvement of this setup can be expected. For example, the adjunction of an antireflection coated end-cap on both end-facets of the rod can not only

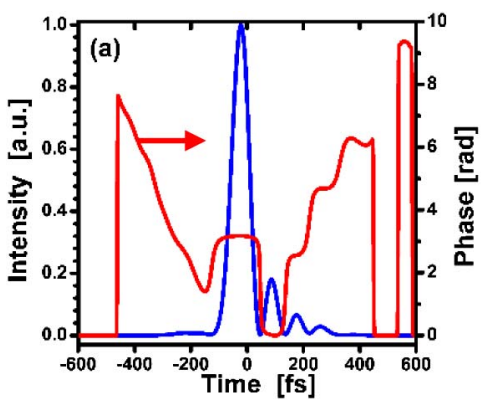

(b)

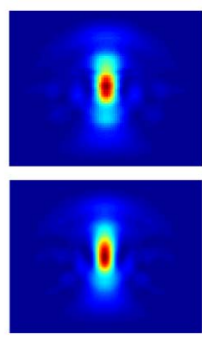

Fig. 5. (a) Retrieved temporal profile from the SHG-FROG measurement. (b) Experimental (top) and retrieved (bottom) FROG trace at maximum energy. 
prevent potential optical damages when approaching the self-focusing limit but also avoid $\sim 7 \%$ Fresnel losses. As already mentioned, pulse compression is currently limited by the efficiency and by the high-order dispersion terms produced by the compressor that both lengthen the pulse full width by $15 \%$, compared to the Fouriertransform-limited pulse duration, and also delocalize $15 \%$ of the energy in it pedestal. Therefore, this setup has the potential to provide pulse peak power well in excess of $100 \mathrm{MW}$ peak power that is sufficient to drive high field physics experiments.

In conclusion, we proposed an architecture for the postcompression of ultrashort pulses that takes advantage of the latest coherent combination strategies. In a proof-of-principle experiment, we demonstrated the energy scaling of a solid-core fiber-based nonlinear temporal compression technique thanks to the implementation of a spatio-temporal coherent combining scheme including highly birefringent crystals and a SI. This tabletop system is able to generate up to 32 replicas of the incident femtosecond laser pulse, dividing the peak power by the same factor. We demonstrated the generation of $7.5 \mu \mathrm{J}, 71 \mathrm{fs}$ pulses at a repetition rate of $100 \mathrm{kHz}$, corresponding to a peak power of $86 \mathrm{MW}$. We also discussed simple strategies to improve the peak intensity well beyond $110 \mathrm{MW}$ with minor modification to this setup. At large nonlinearity levels and numbers of replicas, the appearance of parasitic pulses around the main output pulse is observed, due to imperfect recombination in the time domain, which has been identified as the main limitation of this method. This experiment represents the first use of femtosecond coherent combination outside the scope of optical amplifiers, and shows the applicability of the idea to a wider range of energy- limited ultrafast systems. The authors believe that this technique could, in particular, be advantageously implemented in a high-energy gas-filled capillary postcompression setup to scale the ouput energy of few-cycle pulses toward $10 \mathrm{~mJ}$, well beyond the current ionization limit.

The authors acknowledge the financial support of Agence Nationale de la Recherche through the Multifemto project.

\section{References}

1. C. Jocher, T. Eidam, S. Hädrich, J. Limpert, and A. Tünnermann, Opt. Lett. 37, 4407 (2012).

2. S. Bohman, A. Suda, T. Kanai, S. Yamaguchi, and K. Midorikawa, Opt. Lett. 35, 1887 (2010).

3. C. Fourcade-Dutin, B. Debord, M. Dontabactouny, C. Hönninger, E. Mottay, L. Vincetti, F. Gerome, and F. Benabid, in Conference on Lasers and Electro-Optics, OSA Technical Digest (Optical Society of America, 2013), paper CTh5C.7.

4. S. Roither, A. J. Verhoef, O. D. Mûcke, G. A. Reider, A. Pugzlyz, and A. Baltuska, Opt. Express 20, 25121 (2012).

5. L. Daniault, M. Hanna, D. N. Papadopoulos, Y. Zaouter, E. Mottay, F. Druon, and P. Georges, Opt. Lett. 36, 4023 (2011).

6. S. Zhou, F. W. Wise, and D. G. Ouzounov, Opt. Lett. 32, 871 (2007).

7. Y. Zaouter, L. Daniault, M. Hanna, D. N. Papadopoulos, F. Morin, C. Hönninger, F. Druon, E. Mottay, and P. Georges, Opt. Lett. 37, 1460 (2012).

8. L. Daniault, M. Hanna, D. N. Papadopoulos, Y. Zaouter, E. Mottay, F. Druon, and P. Georges, Opt. Express 20, 21627 (2012).

9. Y. Zaouter, F. Guichard, L. Daniault, M. Hanna, F. Morin, C. Hönninger, E. Mottay, F. Druon, and P. Georges, Opt. Lett. 38, 106 (2013). 